\title{
A near-field scanning optical microscopy study of the photoluminescence from GaN films
}

\author{
Jutong Liu, N. R. Perkins, and M. N. Horton \\ University of Wisconsin-Madison, Madison, Wisconsin 53706 \\ J. M. Redwing and M. A. Tischler \\ Advanced Technology Materials, Inc., Danbury, Connecticut 06810 \\ T. F. Kuech ${ }^{\text {a) }}$ \\ University of Wisconsin-Madison, Madison, Wisconsin 53706
}

(Received 16 July 1996; accepted for publication 24 September 1996)

\begin{abstract}
We have achieved spatially resolved photoluminescence from $\mathrm{GaN}$ films using a near-field scanning optical microscope (NSOM). GaN films grown by hydride vapor phase epitaxy (HVPE) and metal-organic vapor phase epitaxy (MOVPE) on sapphire substrates have been studied. We have performed spatial scans of topography, band edge, and yellow luminescence signals. Atomic force microscopy measurements were also made and compared with the NSOM topography. We have found spatial variations in photoluminescence characteristics at the submicron scale for both HVPE and MOVPE GaN. The observed enhancement of yellow luminescence at multiatomic step edges on the HVPE GaN surface suggests that the yellow luminescence is associated with chemical impurities incorporated during the growth of GaN films. (c) 1996 American Institute of Physics. [S0003-6951(96)00549-9]
\end{abstract}

The successful development of short wavelength light emitting diodes and the most recent realization of nitride semiconductor lasers have stimulated great interests in the applications of this material for blue and ultraviolet optoelectronic devices. ${ }^{2}$ Epitaxial films grown by chemical vapor deposition or molecular beam epitaxy on conventional substrates, such as $\mathrm{Al}_{2} \mathrm{O}_{3}$ or $\mathrm{SiC}$, contain a high density of dislocations, on the order of $10^{8}-10^{10} \mathrm{~cm}^{-2}$. $^{3}$ The extended and point defects in GaN films may greatly impact the performance of many devices. For example, the microscopic inhomogeneity could limit the achievable gain of laser structures. Recently, there have been several reports on the studies of microscopic properties of the GaN materials at different spatial scales. ${ }^{4-6}$ In this letter, we report the high spatial resolution photoluminescence (PL) measurements of $\mathrm{GaN}$ films by near-field scanning optical microscopy (NSOM). ${ }^{7}$ We have performed spatially resolved studies of band edge PL (BL) as well as yellow luminescence (YL). Yellow luminescence is frequency found in GaN materials and has been attributed to both chemical and physical defects. ${ }^{8-11}$ This optical information is correlated with the detailed morphological features obtained simultaneously by NSOM, as well as atomic force microscopy (AFM) measurements. Secondary ion mass spectroscopy (SIMS) measurements were also performed to determine the concentrations of chemical impurities in the GaN films.

The NSOM is a home built system with a commercial electronic control unit. ${ }^{12}$ All the experiments were performed in illumination mode, where the probe served as an excitation source. The $\mathrm{He}-\mathrm{Ne}$ laser light, used for the standard shear force scanning distance regulation. ${ }^{13} \mathrm{~A}$ parabolic reflector is used which has been specifically designed to collect the optical signals with optimum efficiency. The NSOM

${ }^{a)}$ Electronic mail: kuech@engr.wisc.edu probes used in this study were pulled from UV grade single mode optical fibers using a modified commercial fiber puller $^{14}$ and the tips were coated with aluminum. The tip radius and aperture size were determined by low voltage scanning electron microscopy on probes fabricated under similar processing conditions to be $\sim 0.25 \mu \mathrm{m}$ and $\sim 70 \mathrm{~nm}$, respectively. A He-Cd laser, with $325 \mathrm{~nm}$ emission wavelength, was used as the excitation source. All experiments in this study were performed at room temperature. Broad area conventional PL measurements on both hydride vapor phase epitaxy (HVPE) and metal-organic vapor phase epitaxy (MOVPE) samples showed the presence of YL under low excitation power densities. The NSOM-based PL measurements were carried out at a specific position on the sample as a function of wavelength or through spatial scans with fixed collection wavelength. In the latter case, the collection wavelength was set at either the peak of the BL $(365 \mathrm{~nm}$ for HVPE GaN or $364 \mathrm{~nm}$ for MOVPE GaN) or the center of YL band (580 nm for HVPE GaN or $540 \mathrm{~nm}$ for MOVPE $\mathrm{GaN})$. The spectrometer resolution was set to a wavelength resolution of $3 \mathrm{~nm}$. The $\mathrm{BL}$ emission from these samples possessed a full width at half-maximum (FWHM) of $\sim 8 \mathrm{~nm}$ while the YL band was much broader. The full PL spectrum was obtained at multiple points over the surface. No shift in these peak positions or FWHM values was observed over the measurement area. The contrast in NSOM-based PL images is therefore due only to intensity variation in each of the two PL bands and not due to local peak shifts in the PL spectrum.

GaN samples grown on the (0001) plane of sapphire by $\mathrm{HVPE}^{15}$ and MOVPE ${ }^{16}$ were studied. The HVPE growth rate was $\sim 1.5 \mu \mathrm{m}$ per minute and the GaN film thickness was $\sim 180 \mu \mathrm{m}$. The nonintentionally doped HVPE GaN sample was $n$ type with $n \sim 10^{18}-10^{19} \mathrm{~cm}^{-3}$. The MOVPE samples were grown using trimethyl aluminum, trimethyl gallium, and ammonia as precursors, and hydrogen as the carrier gas. $\mathrm{SiH}_{4}\left(1 \mathrm{ppm}\right.$ in $\left.\mathrm{H}_{2}\right)$ was used as an intentional $n$-type dopant 
source. A thin $(15 \mathrm{~nm})$ AlN buffer layer was initially grown on the sapphire substrate at $550^{\circ} \mathrm{C}$. The temperature was then increased to $1100{ }^{\circ} \mathrm{C}$ for growth of a nominally $3-\mu \mathrm{m}$ thick GaN layer. There were three Si doped samples and one nominally undoped sample. Electron concentration of the four samples were $<1 \times 10^{17}$ (not measurable), 2.6 $\times 10^{17}, 2.7 \times 10^{18}$, and $7.0 \times 10^{18} \mathrm{~cm}^{-3}$, determined by Hall measurements.

The concentrations of chemical impurities in the HVPE $\mathrm{GaN}$ were determined by secondary ion mass spectroscopy (SIMS). The average concentrations within the analysis depth $(\sim 3 \mu \mathrm{m})$ for $\mathrm{Si}$ and $\mathrm{O}$ were $1 \times 10^{18}$ and 4 $\times 10^{20} \mathrm{~cm}^{-3}$, respectively. $\mathrm{C}$ was found to be below the detection limit $\left(<6 \times 10^{16} \mathrm{~cm}^{-3}\right)$. The origins of these chemical impurities are presently unknown.

The topographic, BL and YL images collected over the same scan area on the HVPE sample are shown in Fig. 1. The AFM measurements were also performed and compared with the topography obtained by NSOM. Large terraces bounded by steps, several unit cells high, dominate the morphology. Both NSOM and AFM measurements yield an average step height of $3 \mathrm{~nm}$. The average terrace width is 600 $\mathrm{nm}$. The variation of $\mathrm{BL}$ at the submicron spatial scale is clearly observed with no correlation to the surface morphology. On the other hand, the YL and topographic signals are strongly correlated. The YL is clearly seen to be enhanced at the multiatomic step edges. The smallest feature observed in the YL image is $\sim 170 \mathrm{~nm}$.

The NSOM measurements revealed the existence of inhomogeneities in optical properties on a finer scale than previously reported. ${ }^{5}$ Spectral characteristic variations, i.e., different spatial distributions of $\mathrm{BL}$ and $\mathrm{YL}$ signals, were clearly observed with high spatial resolution. Changes in either surface recombination velocity or the local defect or impurity levels could be possible sources of the observed submicron scale spatial variations of BL and YL intensities. The lateral extent of the probed volume in the NSOM-based PL experiment is determined by the probe size, surface recombination velocity, and the diffusion of photogenerated carriers in the semiconductor material. ${ }^{17}$ This lateral resolution, estimated from the PL images, is better than $\sim 200 \mathrm{~nm}$.

Both the YL and BL will be affected by the local changes in the surface recombination through the density of local changes in the minority carrier concentration as well as by their relative radiative and nonradiative transition rates. The YL is derived from transitions involving spatially localized deep level defects. ${ }^{18,19}$ Depending on the relative concentration of the deep levels responsible for the YL and its capture and recombination rates, a wide range of coupling between the BL and YL could be expected. Even though BL and YL are composed of competing and coupled radiative recombination mechanisms, the spatial distributions of these two PL bands are not correlated in the present study. The coupling of the radiative and nonradiative transitions at the surface, as well as in the bulk, is not apparent. This may be attributed to a variation of combined influences, such as low YL-related defect concentration or a large disparity in the radiative lifetimes associated with $\mathrm{BL}$ and $\mathrm{YL}$.

We have observed the enhancement of YL intensity at multiatomic step edges on HVPE GaN surface. BL intensity,

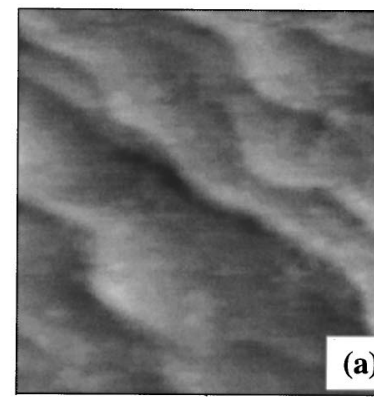

\section{$13 \mathrm{~nm}$

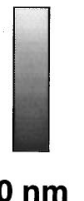

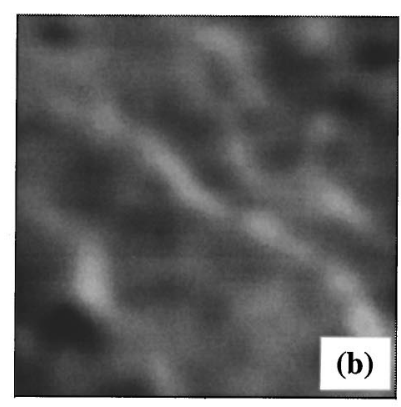

3.07 au

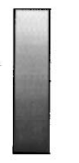

3.04 au

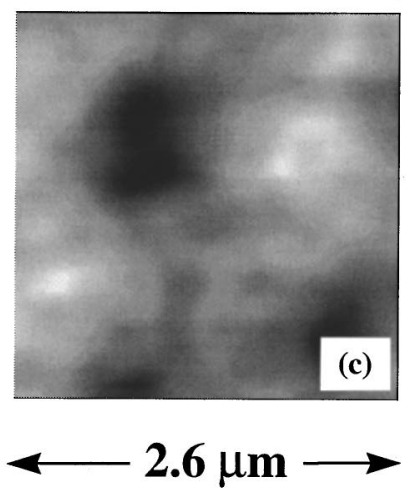

2.2 au

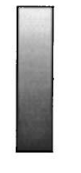

2.1 au

FIG. 1. Topographic (a), yellow luminescence (b), and band edge luminescence (c) images of HVPE GaN collected over the same scan area $(2.6$ $\mu \mathrm{m} \times 2.6 \mu \mathrm{m})$. The total variations of the $\mathrm{YL}$ and $\mathrm{BL}$ are $1 \%$ and $5 \%$, respectively. A strong correlation exists between the large terrace structure and YL spatial distribution, i.e., YL is enhanced at the multiatomic step edges.

on the other hand, is not correlated with the step structure. This fact strongly suggests that a higher density of deep luminescence centers near step edges within the NSOM sampling volume, instead of a local change in surface recombination velocity, is the source of YL variation in HVPE GaN. The deep defects responsible for YL in GaN material have been suggested to be chemical impurities ${ }^{8-10}$ or native defects. ${ }^{10}$ In many growth systems, step edges serve as active sites for impurity incorporation. ${ }^{20}$ The probability of foreign atoms being incorporated into the crystal is higher at the strongly stepped riser (step edge) portion of the terrace than on the smooth terrace tread. Impurities can be trapped and incorporated in the terrace riser traces in the layer as the terraces migrate from the substrate to the surface in certain growth modes. ${ }^{21} \mathrm{Si}$ and $\mathrm{O}$ are found by SIMS measurements to be present in the HVPE GaN sample at concentrations of $\sim 10^{18}$ and $\sim 10^{20} \mathrm{~cm}^{-3}$, respectively. The enhancement of YL at step edges in HVPE GaN could be related to the enhanced incorporation of these chemical impurities at or near multiatomic step edges often seen in a variety of growth systems. 


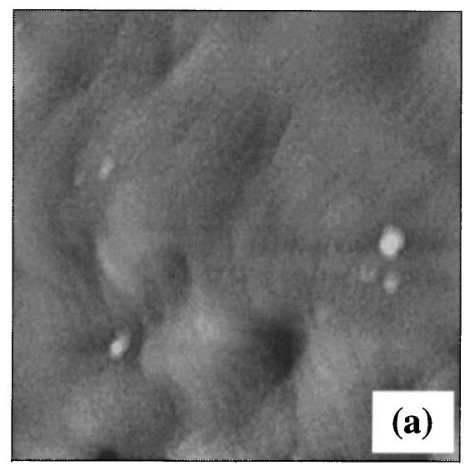

\section{$11 \mathrm{~nm}$

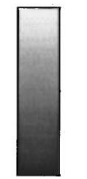 \\ $\mathbf{0} \mathbf{n m}$}

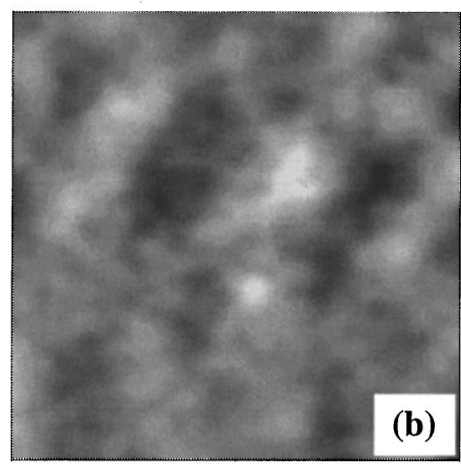

$1.92 \mathrm{au}$

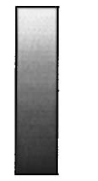

1.86 au



$5.2 \mu \mathrm{m}$

FIG. 2. Topographic (a), yellow luminescence (b), and band edge luminescence (c), images of the Si-doped MOVPE GaN sample (7.0 $\left.\times 10^{18} \mathrm{~cm}^{-3}\right)$ collected over the same scan area $(5.2 \mu \mathrm{m} \times 5.2 \mu \mathrm{m})$. The total variations of the $\mathrm{YL}$ and $\mathrm{BL}$ are $3 \%$ and $1 \%$, respectively. No correlation is observed between these signals. Terraces and steps are much smaller on MOVPE GaN than HVPE GaN. Submicron scale variations of band edge and YL intensities are indicated by the quantitative lateral correlation length analysis, i.e., the lateral correlation lengths for the YL and BL images are 0.27 and $0.42 \mu \mathrm{m}$, respectively.

We have also performed NSOM scans on the MOVPE $\mathrm{GaN}$ samples and found much different surface morphology than the HVPE sample. The topographic, YL and BL images collected over the same scan area on the Si-doped MOVPE sample $\left(7.0 \times 10^{18} \mathrm{~cm}^{-3}\right)$ are shown in Fig. 2. These images were analyzed to determine the lateral correlation length and root mean square (rms) roughness which characterize these surfaces. The lateral correlation length is a quantitative measure of the smallest feature size on an image. ${ }^{22}$ The calculated lateral correlation lengths, included in the figure cap- tion, indicate submicron variations of the optical signals. On MOVPE GaN, the terraces are much narrower than the ones on HVPE GaN. We have performed NSOM measurements on all four MOVPE samples with submicron scale spatial variations in BL and YL intensities clearly observed. However, the correlation between YL spatial distribution with step morphology could not be investigated in detail due to the high step density and the NSOM resolution limits.

In conclusion, we have studied the photoluminescence from HVPE and MOVPE GaN films with very high spatial resolution. The variations of photoluminescence characteristics at submicron spatial scale are clearly observed. The examination of the detailed correlation between morphology, band edge, and YL suggests that YL in HVPE GaN could be associated with defects, most probably chemical impurities, incorporated at step edges of both atomic and multiatomic steps.

This work was supported by Army Research Office (DAAH04-95-1-0322), the National Science Foundation through the Materials Research Group on Chemical Vapor Deposition (MRG DMR9121074), and ARPA URI on Visible Light Emitters. The authors would like to acknowledge assistance from Dr. Patrick Moyher, Kirk J. Puotinen, and Nicole M. Pauly.

${ }^{1}$ H. Amano, M. Kito, K. Hiramatsu, and I. Akasaki, Jpn. J. Appl. Phys. 28, L2112 (1989).

${ }^{2}$ S. Nakamura, M. Senoh, S. Nagahama, N. Iwasa, T. Yamada, T. Matsushita, H. Kiyoku, and Y. Sugimoto, Jpn. J. Appl. Phys. 35, L74 (1996).

${ }^{3}$ See for example, F. A. Ponce, B. S. Krusor, J. S. Majors, Jr., W. E. Plano, and D. F. Welch, Appl. Phys. Lett. 67, 410 (1995).

${ }^{4}$ W. Qian, G. S. Rohrer, M. Skowronski, K. Doverspike, L. B. Rowland, and D. K. Gaskill, Appl. Phys. Lett. 67, 2284 (1995).

${ }^{5}$ F. A. Ponce, D. P. Bour, W. Gotz, and P. J. Wright, Appl. Phys. Lett. 68, 57 (1995).

${ }^{6}$ B. Garni, Jian Ma, N. Perkins, Jutong Liu, T. F. Kuech, and M. G. Lagally, Appl. Phys. Lett. 68, 1331 (1996).

${ }^{7}$ R. D. Grober, T. D. Harris, J. K. Trautman, E. Betzig, W. Wegscheider, L. Pfeiffer, and K. West, Appl. Phys. Lett. 64, 1421 (1994).

${ }^{8}$ J. I. Pankove and J. A. Hutchby, J. Appl. Phys. 47, 5387 (1976).

${ }^{9}$ P. Hacke, A. Maekawa, N. Koide, K. Hiramatsu, and N. Sawaki, Jpn. J. Appl. Phys. 33, 6443 (1994).

${ }^{10}$ X. Zhang, P. Kung, D. Walker, A. Saxler, and M. Razeghi, Mater. Res. Soc. Symp. Proc. 395, 425 (1996).

${ }^{11}$ J. Neugebauer and C. G. Van de Walle, Appl. Phys. Lett. 69, 503 (1996).

${ }^{12}$ TopoMetrix Corporation, 5403 Betsy Ross Drive, Santa Clara, CA 950541192.

${ }^{13}$ E. Betzig, P. L. Finn, and J. S. Weiner, Appl. Phys. Lett. 60, 2484 (1992).

${ }^{14}$ Sutter Instrument Company, 40 Leveroni Court, Novato, CA 94949.

${ }^{15}$ N. R. Perkins, M. N. Horton, Z. Z. Bandic, T. C. McGill, and T. F. Kuech, Mater. Res. Soc. Symp. Proc. 395, 243 (1996).

${ }^{16} \mathrm{~J}$. M. Redwing and M. A. Tischler (unpublished).

${ }^{17}$ Jutong Liu and T. F. Kuech, Appl. Phys. Lett. 69, 662 (1996).

${ }^{18}$ T. Ogino and M. Aoki, Jpn. J. Appl. Phys. 19, 2395 (1980).

${ }^{19}$ E. R. Glaser, T. A. Kennedy, K. Doverspike, L. B. Rowland, D. K. Gaskill, J. A. Freitas, Jr., M. Asif Khan, D. T. Olson, J. N. Kuznia, and D. K. Wickenden, Phys. Rev. B 51, 13326 (1995).

${ }^{20}$ B. Fischer, E. Bauser, P. A. Sullivan, and D. L. Rode, Appl. Phys. Lett. 33, 78 (1978).

${ }^{21}$ E. Bauser and G. A. Rozgonyi, Appl. Phys. Lett. 37, 1001 (1980).

${ }^{22}$ H.-N. Yang, G.-C. Wang, and T.-M. Lu, Diffraction from Rough Surfaces and Dynamic Growth Fronts (World Scientific, Singapore, 1993), Chap. II. 